\title{
UNA EPIDEMIOLOGÍA SOCIAL PARA AMÉRICA LATINA: UNA NECESIDAD MÁS ALLÁ DE LA REFLEXIÓN SOBRE LAS INEQUIDADES EN SALUD
}

\author{
A SOCIAL EPIDEMIOLOGY FOR LATIN AMERICA: THE NEED TO GO BEYOND JUST THINKING ABOUT \\ HEALTH INEQUITIES
}

Jay S. Kaufman ${ }^{1, a, b}$, Edward Mezones-Holguín 2,3,b,c

La Epidemiología es el estudio de las relaciones entre exposiciones y enfermedades a nivel poblacional, mientras que la Epidemiología Social constituye un subcampo dentro de la Epidemiología, el cual se caracteriza por centrar su estudio en las exposiciones que, de alguna manera, están vinculadas a un estado, posición o rango social (1). Ejemplos típicos de estado social o marcadores de roles sociales son el sexo/ género, raza/etnicidad y la posición/clase socioeconómica. Debido a que la Epidemiología es una ciencia observacional poblacional, por lo general las variables sociales y demográficas siempre cumplen un rol en el diseño o análisis; sin embargo, en el enfoque de la Epidemiología Social estas variables son estudiadas como exposiciones primarias. Asimismo, existe en la Epidemiología Social una tendencia natural a confiar en la teoría social para entender los procesos etiológicos en estudio, puesto que el significado de estas medidas es revelado por los enfoques sociológicos, permitiéndonos entender cómo las personas logran alcanzar diferentes niveles de estatus dentro de la sociedad y observar las consecuencias de estar en una categoría frente a otra ${ }^{(2)}$.

Las dos actividades primarias en la Epidemiología son la vigilancia y la investigación etiológica, ambas también son esenciales dentro de la Epidemiología Social. En la vigilancia, el propósito es describir el mundo como realmente es; en el contexto de la Epidemiología Social, esto significa representar los patrones de la enfermedad a lo largo de las dimensiones de la distinción social, tales como las tasas o los riesgos de diferentes desenlaces por categorías de variables como la educación, los ingresos o la etnia. Esta actividad requiere un mínimo de ajustes, ya que el propósito es describir el mundo real, no un mundo hipotético. Por tanto, la vigilancia podría necesitar el modelamiento leve de algunos aspectos dispersos, o dar cuenta de los errores en la información obtenida, pero no está sujeta al análisis de confusión o a otras nociones de causalidad, ya que la finalidad no es estimar los efectos de las exposiciones, sino simplemente presentar la distribución del desenlace a través de algunas de las dimensiones sociales relevantes. A este nivel, la interpretación de un patrón como una inequidad o injusticia es una cuestión de valores sociales que va más allá de la cuestión objetiva de lo "¿qué es?" e implica un cuestionamiento más profundo: "¿qué debería ser?" (3).

En contraste, la investigación etiológica no se ampara en el mundo real, por el contrario, yuxtapone el mundo real frente a un mundo hipotético, focalizándose en entender la relación entre una exposición y un desenlace determinados. En ese marco, la relación causal es el cambio esperado en el desenlace después de una intervención sobre la exposición. Partiendo de que la Epidemiología es una ciencia observacional, realmente no es posible cambiar intencionalmente la exposición; no obstante, podemos ver la variación que ocurre naturalmente en la exposición, vincular ello a los cambios observados en los desenlaces. La intención, por consiguiente, no es describir el mundo real sino, en su lugar, estimar qué pasaría, hipotéticamente, si intervenimos en dicho mundo. En el contexto epidemiológico social, esto podría ser la estimación de los efectos de los suplementación sobre los ingresos familiares, los programas educativos y otras intervenciones

Department of Epidemiology, Biostatistics, and Occupational Health. McGill University. Montreal, Canada.

Centro Nacional de Salud Pública, Instituto Nacional de Salud. Lima, Perú.

Escuela de Medicina, Universidad Peruana de Ciencias Aplicadas. Lima, Perú.

Editor invitado; ${ }^{\mathrm{b}} \mathrm{PhD}$ en Epidemiología; ${ }^{\mathrm{c}}$ médico cirujano, maestro en Ciencias en Epidemiología Clínica.

Recibido: 26-11-2013 Aprobado: 27-11-13

Citar como: Kaufman JS, Mezones-Holguin E. Una epidemiología social para América Latina: una necesidad más allá de la reflexión sobre la inequidad en salud [editorial]. Rev Peru Med Exp Salud Publica. 2013;30(4):543-6. 
sociales. Si pudiéramos diseñar y conducir un ensayo aleatorizado de una intervención de este tipo, no habría la necesidad de utilizar a la Epidemiología Social para deducir el efecto causal. No obstante, lo real es que muchos de estos datos se obtienen de manera pasiva, por tanto, para confiar en ellos el desafío de la Epidemiología Social es modelar estas observaciones de manera inteligente, de modo que pueda permitirnos obtener el impacto de las acciones, cuando en realidad estas no fueron realizadas. No obstante, a pesar de las profundas dificultades que se encuentran en la puesta en marcha de este tipo de ejercicio, la mayor parte de trabajos publicados en Epidemiología Social se encuentran dentro de esta categoría ${ }^{(4)}$.

La mayor innovación en la Epidemiología Social en la última década ha sido la de tomar seriamente los desafíos planteados en la investigación etiológica. Debido a los efectos causales se definen como los contrastes entre los resultados potenciales bajo diferentes intervenciones, estos no pueden ser observados fuera del contexto de un ensayo aleatorizado, es por ello que este tipo de estudio ha logrado una alta notoriedad convirtiéndose en una especie de "estándar de oro" (gold standar) para el estudio causal, más aun cuando están ligados a la teoría del contrafactual (counterfactual theory). Los ensayos aleatorizados utilizan cambios reales en las exposiciones para responder a la pregunta sobre cuáles resultados se observaron en diferentes intervenciones de política social. Sin embargo, cuando nos limitamos a los datos observacionales, nos enfrentamos siempre al problema de que los sujetos que tienen una exposición, y aquellos que no la tienen, pueden ser diferentes en otras características que no han sido medidas. A este término de desequilibrio se le denomina "confusión", y constituye una de las principales amenazas para la validez de la Epidemiología Social observacional. Un punto de vista moderno para enfrentar este problema requiere de una estrategia de identificación, la cual precisa de aproximaciones tanto en el diseño como en el análisis, que puedan mitigar de manera convincente el desequilibrio generado por la selección en el tratamiento por factores no medidos. En Epidemiología Social, estas competencias incluyen varios tipos de técnicas de pareamiento (matching), el uso de grupos control, y el descubrimiento inteligente de las variables instrumentales o la discontinuidad en la regresión (regression discontinuities) ${ }^{(5,6)}$.

Consecuentemente, la Epidemiología Social ha madurado en gran medida, yendo desde una larga colección de estudios de asociación hacia una subdisciplina en la que hay un intento serio de informar en el marco de las políticas sociales. Muchas políticas potenciales apuntan a impactar en la educación, el ingreso y la riqueza, las implicaciones socioeconómicas o raza/etnicidad y el género. Estas son exposiciones epidemiológicas clásicas, y en la medida que las posibles políticas sociales tengan como propósito mejorar la salud pública, la Epidemiología Social se convierte en el campo encargado de proveer a los tomadores de decisiones de la información científica necesaria para elegir de manera racional entre las opciones de política ${ }^{(7)}$.

El alcance de la Epidemiología Social moderna es extenso, abarca todas las etapas del ciclo vital, todas las formas de enfermedades y daños; asimismo, recurre a una amplia gama de medidas y conceptos. Existe un esfuerzo intelectual considerable dirigido a la conceptualización y estimación de la posición social, así como a la medición de conceptos relacionados como pobreza y discriminación. Del mismo modo, en esta subdisciplina existe una inversión concertada para modelar la desigualdad y descomponer varias medidas de inequidad con el objeto de lograr un mejor entendimiento de cómo las enfermedades se distribuyen en la sociedad. Las comunidades y los barrios son un importante foco de estudio en la Epidemiología Social, puesto que los seres humanos viven en maneras que son conscientemente determinadas, socialmente segregadas y políticamente sensibles. Esta situación requiere frecuentemente un modelamiento espacial y multinivel, cuando los datos observados en múltiples niveles jerárquicos de la organización están disponibles para su estudio. Asimismo, este set de preguntas de investigación han permitido la puesta en marcha de los análisis de red y otros enfoques que enfatizan en la interconexión entre las personas como un principio central de la vida social, los cuales tienen profundas implicaciones para la salud ${ }^{(8,9)}$.

Como una ciencia social observacional, la Epidemiología Social cae naturalmente bajo la influencia del trabajo propio de la Sociología, la Economía, y la Geografía. La "polinización cruzada" (cross-pollination) de otras disciplinas ha sido el mayor motor en la innovación en diseño y análisis, y muchas de las "nuevas" ideas en este campo son meramente tomadas de otras partes. Indudablemente, el futuro vislumbra un intercambio interdisciplinario más intenso, y una creciente influencia de otras ramas de la Epidemiología, incluyendo las aproximaciones moleculares y genéticas. En ese sentido, es preciso mencionar que la antigua demarcación de los límites disciplinarios se está derrumbando, y cada vez resulta mucho más difícil circunscribir el territorio de una subdisciplina frente a otra. Subsecuentemente, la presión sobre los jóvenes epidemiólogos sociales es poseer un acervo cada vez más extenso de habilidades para el diseño y análisis, y estar familiarizado con una amplia y sustantiva base teórica. Considerando que la enfermedad emerge fundamentalmente de un desequilibrio o déficit, y dado que los seres humanos tienen experiencias sociales fascinantemente complejas, las formas como pueden ocurrir estos desequilibrios son prácticamente infinitas. Por consiguiente, el gran 
desafío de la Epidemiología Social se encuentra en posicionarse de manera estratégica para hacer frente a toda la miríada de vías por las cuales la enfermedad opera a través de los mecanismos sociales.

En las últimas décadas, Latinoamérica (LA), ha presentado cambios substanciales en materia económica, social y demográfica; con intensificación en algunos casos de las diferencias culturales y étnicas. Estas variaciones no han sido uniformes en la región, situación que, en su conjunto, ha generado diversificaciones entre los países y dentro de ellos, con implicaciones relevantes en la salud pública. En este contexto, la investigación en Epidemiología Social cumple un rol protagónico no solo en la identificación de los elementos que podrían caracterizar las poblaciones más desfavorecidas y su asociación con la enfermedad $u$ otros desenlaces, sino también en la constitución de estrategias para el direccionamiento de políticas en salud. Al respecto, en LA se ha descrito, por ejemplo, que las percepciones en salud se ven afectadas por los patrones culturales y las variables económicas agregadas ${ }^{(10)}$; se ha evidenciado la asociación entre el capital social y los estados de salud (11); y se ha explorado la necesidad de la utilización de los factores relacionados al trabajo para explicar las desigualdades y la medición de la posición socioeconómica en la zona ${ }^{(12)}$. Además, se ha puntualizado que las aproximaciones de salud pública son variables en la región, aunque se está teniendo un importante esfuerzo para la actuar sobre los determinantes sociales de la salud (13) y por disminuir el "divorcio" entre la academia y los tomadores de decisiones ${ }^{(14)}$. No obstante, las fuerzas para el desarrollo de la investigación epidemiológica difieren por país, encontrándose incluso discrepancias entre ellos (15); por lo que se sugiere la puesta en marcha de trabajo colaborativo interinstitucional y multidisciplinario dentro de lo países, así como entre ellos, incluyendo instancias fuera de LA.

Si bien resulta reducido el espacio para brindar un panorama exhaustivo sobre la Epidemiología Social, y en particular acerca del estudio de las inequidades en salud en LA, es suficiente para reconocer la necesidad de contar con una aproximación desde la perspectiva de este subcampo de la Epidemiología; más aun cuando el carecer de investigaciones en el área de Epidemiología Social podrían constituir, a su vez, un factor contribuyente en la acentuación de los problemas de salud en la región. En ese sentido, la RPMESP como órgano de difusión científica de vanguardia en la salud pública LA, ha dedicado el presente número a la Epidemiología Social y al estudio de las inequidades en salud. En este marco se presenta un simposio, el cual incorpora una interesante perspectiva sobre el crecimiento económico y la inequidad en salud desarrollado por el profesor Tapia Granados del Institute for Social Research de la
University of Michigan, quien a través de un recuento histórico describe que, paradójicamente, en periodos en que la economía crece, las desigualdades tienden a acentuarse. Asimismo, los investigadores Frenz y Titelman, de la Escuela de Salud Pública de la Universidad de Chile y de la Comisión Económica para América Latina (CEPAL), respectivamente, exponen a la equidad en salud como un paso importante en la búsqueda de la igualdad en el contexto de la redemocratización en LA; consideran que el rol de los gobiernos es fundamental en dicho proceso, requiriendo el fortalecimiento de las capacidades a diversos niveles, con un trabajo de interacción entre múltiples instituciones y sectores. Por su parte, el equipo interinstitucional liderado por el profesor Haro-García del Departamento de Salud Pública de la Universidad Nacional Autónoma de México, precisa que las condiciones laborales conforman un componente cardinal para la generación de inequidades en salud, sugiriendo que las mejoras a dicho nivel con la participación estatal correspondiente contribuirán a la igualdad y bienestar social. El campo de las enfermedades infecciosas es afrontado por Vanlerberghe y Verdonck, investigadoras del Institute of Tropical Medicine Antwerp, quienes exponen el caso del dengue como un marcador de inequidad en salud, proponen mejoras en la prevención, control y manejo e incorporando los condicionantes sociales de la enfermedad. Contamos también con el aporte del académico peruano Sánchez-Moreno quien desde su experiencia como presidente emérito de la Academia Peruana de Salud, manifiesta su perspectiva sobre los factores que han contribuido a la generación de las inequidades en salud en Perú y muestra reflexiones acerca de las estrategias necesarias hacia una política de Estado para una salud más equitativa.

Asimismo, en la sección de revisión las profesoras Fleischer y Diez-Roux del Center for Research in Nutrition and Health Disparities de la University of South California y del Departamento de Epidemiología de la University of Michigan, describen que la evidencia empírica respecto al estudio de las inequidades en salud en enfermedad cardiovascular es limitada en LA, destaca la necesidad de investigación científica y las implicaciones de ello sobre las políticas de prevención. En la sección especial, se presenta uno de los primeros artículos metodológicos publicados en la RPMESP, desarrollado por un equipo de la Escuela de Salud Pública de Chile y del Departamento de Epidemiología, Bioestadística y Salud Ocupacional de Mcgill University, en este manuscrito, Orellana et al., detallan los conceptos de interacción, sinergia y antagonismo en estudios prospectivos en Epidemiología utilizando para ello herramientas aplicativas. Es importante resaltar, el aporte del catedrático Salaverry García con un ensayo sobre la construcción histórica de la inequidad en salud; en el cual se 
exhibe la evolución y los debates relacionados al concepto de equidad a través del tiempo, ofreciendo también la uniformización de algunas definiciones elementales en el tópico. Dentro de los estudios originales publicados en este número, destacan tres por su cercanía a la temática en cuestión: Jaramillo-García et al., presentan las brechas regionales de la mortalidad infantil en Colombia donde el aseguramiento, la disponibilidad de camas y el acceso a atención médica tienen un rol determinante; por su parte, Castañeda-Hernández et al., notifican la asociación inversa entre la frecuencia de tuberculosis y el índice de desarrollo humano en 165 países del mundo; y Alcázar et al., describen el impacto económico de la desnutrición infantil en Perú, la cual implicaría costos superiores al $2 \%$ del Producto Bruto Interno del país, con diferencias en la frecuencia por regiones geográficas.

Finalmente, invitamos a leer crítica y juiciosamente lo expuesto en este número, agradecemos todas las aportaciones recibidas y estimulamos la remisión de nuevas contribuciones científicas sobre Epidemiología Social e inequidad en salud, un tópico que, como hemos mencionado, resulta imprescindible en la actual agenda científica latinoamericana.

\section{REFERENCIAS BIBLIOGRÁFICAS}

1. Kaufman JS. Social epidemiology. In: Rothman KJ, Greenland S, Lash TL, editors. Modern epidemiology. Philadelphia: Lippincott Williams \& Wilkins; 2008. p. 532-48.

2. Krieger N. Epidemiology and the People's Health: Theory and Context. New York: Oxford University Press; 2011.

3. Harper S, King NB, Meersman SC, Reichman ME, Breen N, Lynch J. Implicit value judgments in the measurement of health inequalities. Milbank Q. 2010;88(1):4-29.

4. Oakes JM, Kaufman JS. Introduction: Advancing methods in Social Epidemiology. In: Oakes JM, Kaufman JS, editors. Methods in Social Epidemiology. San Francisco, CA: John Wiley \& Sons; 2006. p. 3-20.

5. Harper S, Strumpf EC. Social epidemiology: questionable answers and answerable questions. Epidemiol Camb Mass. 2012;23(6):795-8.

6. Dekkers OM. On causation in therapeutic research: observational studies, randomised experiments and instru- mental variable analysis. Prev Med. 2011;53(4-5):239-41.

7. Kaufman JS, Harper S. Health equity: Utopian and scientific. Prev Med. 2013;57(6):739-40

8. El-Sayed AM, Scarborough P, Seemann L, Galea S. Social network analysis and agent-based modeling in social epidemiology. Epidemiol Perspect Innov EPI. 2012;9(1):1

9. Galea $S$, Link BG. Six paths for the future of social epidemiology. Am J Epidemiol. 2013;178(6):843-9.

10. Lora E. Health perceptions in Latin America. Health Policy Plan. 2012;27(7):555-69.

11. Kripper CE, Sapag JC. Capital social y salud en América Latina y el Caribe: una revisión sistemática. Rev Panam Salud Pública Pan Am J Public Health. 2009;25(2):162-70.

12. Muntaner C, Rocha KB, Borrell C, Vallebuona C, Ibáñez C, Benach J, et al. Clase social y salud en América Latina. Rev Panam Salud Pública Pan Am J Public Health. 2012;31(2):166-75.
13. Solar O, Irwin A. Social determinants, political contexts and civil society action: a historical perspective on the Commission on Social Determinants of Health. Health Promot J Aust Off J Aust Assoc Health Promot Prof. 2006;17(3):180-5.

14. Mezones-Holguín E. Health economic evaluations: bringing together academia and policy. Rev Peru Med Exp Salud Pública. 2011;28(3):410-3.

15. Barreto SM, Miranda JJ, Figueroa JP, Schmidt MI, Munoz S, Kuri-Morales PP, et al. Epidemiology in Latin America and the Caribbean: current situation and challenges. Int J Epidemiol. 2012;41(2):557-71.

Correspondencia: Jay S. Kaufman, Ph.D Dirección: Department of Epidemiology, Biostatistics, and Occupational Health, McGill University, 1020 Pine Ave West, Montreal, Quebec H3A 1 A2 CANADA

Teléfono: 514-398-7341

Correo-electrónico:jay.kaufman@mcgill.ca 